\title{
Optical limiting efficiency of an electroactive bis-iminopyridine ligand and its zinc complex
}

\author{
M. Hjiri, ${ }^{1}$ I. Guezguez, ${ }^{1,2}$ K. Iliopoulos, ${ }^{2}$ A. El-Ghayoury, ${ }^{2}$ \\ H. Belmabrouk, ${ }^{1,3}$ M.A. Karpierz, ${ }^{4}$ and B. Sahraoui ${ }^{\dagger 2}$ \\ ${ }^{1}$ Laboratoire d'Electronique et Microélectronique, University of Monastir, Tunisia \\ ${ }^{2}$ LUNAM Université, Université d'Angers, CNRS UMR 6200, Laboratoire MOLTECH-Anjou, \\ 2 bd Lavoisier, 49045 Angers Cedex, France \\ ${ }^{3}$ Department of Physics, College of Science AlZulfi, Majmaah University, KSA \\ ${ }^{4}$ Faculty of Physics, Warsaw University of Technology, Koszykowa 75, 00-662 Warszawa, Poland
}

Received March 7, 2016; accepted March 24, 2016; published March 31, 2016

\begin{abstract}
An electroactive based bis-iminopyridine ligand has been synthesized by a condensation reaction between (4-(6,7-dimethyldithiotetrathiafulvalene)-aniline) with 2,6-diformylpyridine. The complexation of this ligand with $\mathrm{ZnCl}_{2}$ afforded a tetrahedral neutral Zinc metal complex. Nonlinear optical measurements of these structures have given good results in a picosecond regime. The nonlinear absorption of the ligand was significantly enhanced upon complexation with $\mathrm{ZnCl}_{2}$. This prompted us to conduct an experiment of optical limiting at wavelength $532 \mathrm{~nm}$. The nonlinear absorption properties of these structures were studied and compared to the reference material $\mathrm{C}_{60}$.
\end{abstract}

Intensive studies of nonlinear optics (NLO) have been induced by expected applicability of their results in optics, telecommunications and information processing. In this regard, $\pi$-conjugated chromophores reveal good processibility, electronic and optical properties [1-4] including intrinsic hyperpolarizability of 3dicyanomethylene-5,5-dimethyl-1-[2-(4-hydroxyphenyl) ethenyl]-cyclohexene nanocrystallites incorporated into the photopolymer matrices with high third order optical properties [5]. The delocalized $\pi$-electrons give rise to ultrafast response times and large optical nonlinearity, which makes these materials attractive candidates for optoelectronic and photonic applications in optical computing, logic devices, optical switching and organic solar cells [6-9]. We were interested in the $\pi$-conjugated "Push-pull" organic chromophores containing a tetrathiafulvalene electron donating group and an iminopyridyl accepting fragment that can also act as a coordinating unit. This type of compound is of great interest since it gives the possibility to obtain multifunctional materials which may represent an interaction between two or more physical properties, such as magnetism and conductivity [11-13]. Ligands such as

\footnotetext{
*E-mail: guezguezimen@yahoo.fr

${ }^{\dagger}$ E-mail: bouchta.sahraoui@univ-angers.fr
}

iminopyridines have been chosen because of their ability to form a multitude of stable complexes and were, for example, used as catalysts for olefin polymerization [1415]. In this work, we report on the optical limiting properties of an azo-based iminopyridine ligand and its associated zinc(II) complex.

The experiments were investigated by means of the Zscan technique using 16ps mode-locked Nd:YAG lasers operating at $532 \mathrm{~nm}$ at $10 \mathrm{~Hz}$. The details of the Z-scan technique and the procedure used for the determination of the NLO parameters have been described in details elsewhere [7]. Their nonlinear refractive and absorptive properties were measured and are compared with those of the $\mathrm{C}_{60}$ fullerene.

Different concentrations were investigated in order to study the effect of varying the concentration in the low and high excitation energy. Four concentrations $(0.5 \mathrm{mM}$, $1 \mathrm{mM}, 2 \mathrm{mM}$ and $1.5 \mathrm{~mm}$ ) were prepared for the ligand and the corresponding zinc(II) complex.

The structure of the ligand (1) is composed of an extended $\pi$-conjugated system that incorporates tetrathiafulvalene groups and a bis-iminopyridyl fragment. This composition leads to the establishment of an intramolecular charge transfer process between the donor groups and the accepting unit. This behavior makes these systems interesting in terms of nonlinear optical properties. Figure 1 shows the chemical structure of the ligand (1) and of the zinc(II) complex (2).

We started the experimental study exploring the field of linear absorption. For solubility reasons, dimethylformamide (DMF) was used as a solvent. We used a spectrophotometer UV-VIS-IR (Perkin Elmer Lambda 19) in the range of wavelengths between 250 and $800 \mathrm{~nm}$ [11].

The UV-Visible spectrophotometric titration studies of 
the ligand, by increasing the amount of $\mathrm{ZnCl}_{2}$, confirm the existence of single complex specie in the solution.
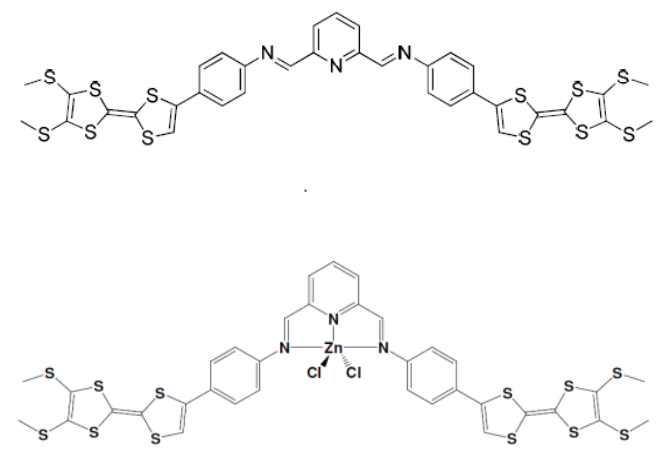

Fig. 1. Chemical structures of the ligand (1) and the complex (2).

The linear absorption spectra illustrate absorption bands in the near-ultraviolet light (in the region of highenergy) with a wavelength between $250 \mathrm{~nm}-300 \mathrm{~nm}$ for the ligand and the complex (Fig. 2). These absorption bands are characteristic of $\pi-\pi^{*}$ and $n-\pi^{*}$ transitions. The absorption band in the visible region is characteristic of intramolecular charge transfer (ICT) between the TTF fragment and iminopyridine unit. Upon complexation with $\mathrm{ZnCl}_{2}$, this ICT undergoes a bathochromic shift indicating an increase of the accepting ability of the coordinated iminopyridyl fragment. This is due to the fact that $\mathrm{ZnCl}_{2}$ is acting as Lewis acid. In addition, the shoulder appearing around $400 \mathrm{~nm}$ is characteristic of a conformational change of the pyridyl fragment (from cisoid to transoid) upon complexation.

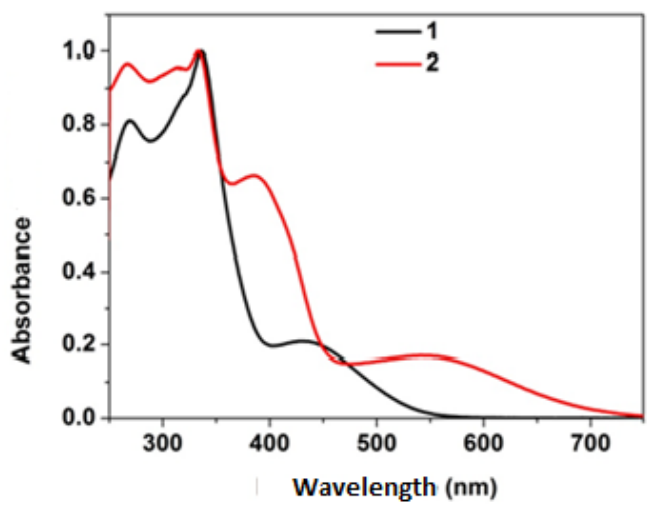

Fig. 2. Electronic absorption spectra of ligand 1 and complex 2.

Interested in the domination of the strong nonlinear absorption of the nonlinear optical response of both compounds 1 and 2, we performed measurements of optical limiting. For the purposes of this experiment, we used the same laser system at $532 \mathrm{~nm}$. Our goal was to compare the efficiency of the optical limiting properties of molecules 1 and 2 with the $\mathrm{C}_{60}$ fullerene molecule, which is well known as a good optical limiter [16-17]. For this reason and to enable a comparison of the three structures, we prepared three solutions having the same linear transmission in the wavelength of laser excitation. The fullerene $\mathrm{C}_{60}$ was dissolved in toluene, whereas compounds 1 and 2 were dissolved in DMF. The absorption spectra of the studied samples shown in Fig. 3 display the same linear absorbance at $532 \mathrm{~nm}$.

Optical limitation was studied as described in the experimental section and in Fig. 3. For each sample, we measured the energy transmitted as a function of incident laser beam energy.

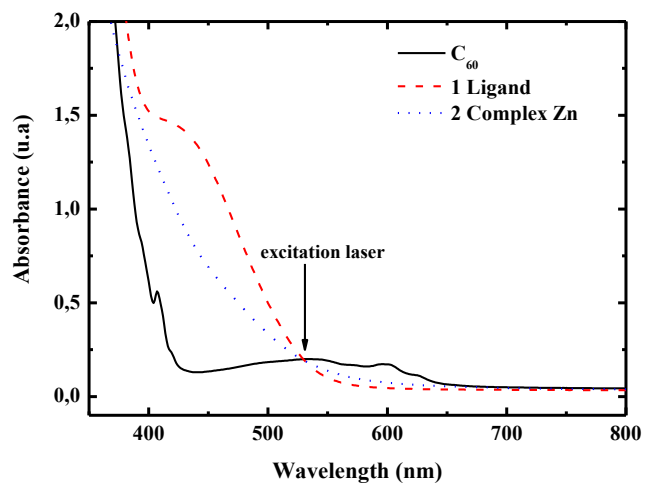

Fig. 3. Absorption spectra for measurements of the optical limitation solutions having the same coefficient of linear transmission at $532 \mathrm{~nm}$.

To determine nonlinear refraction and absorption of the samples, several Z-scan measurements of different concentration solutions were performed at various incident laser energies. All "divided" Z-scans were found to exhibit a transmission peak followed by a post-focal minimum (i.e., a peak-valley configuration) [6], indicative of defocusing behavior of the solutions while buffered water did not exhibit any NLO response for the laser intensities used. From the difference of the measured normalized transmission among the peak and the valley, the so-called $\Delta \mathrm{T}_{\mathrm{P}-\mathrm{V}}$, the nonlinear refractive index parameter $\gamma^{\prime}$ and the real part of $\chi^{(3)}$ were deduced.

Optical confinement was studied as described in the experimental section and in Fig. 4. For each sample, we measured energy transmitted as a function of incident laser beam energy. In Fig. 4 we can see the action of optical limiting of our molecules, which is less than that present in the results. It is obvious that the optical limiting of our molecules is less than that of the reference molecule $\mathrm{C}_{60}$. By comparing the restriction of the optical behavior of the molecules 1 and 2 , it is evident that the complex $\mathrm{Zn}$ is much less effective than the free ligand. 


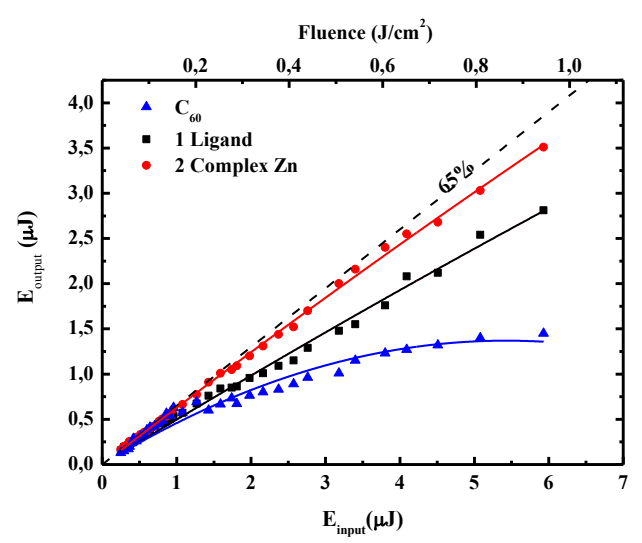

Fig. 4. Optical limiting curves corresponding to the molecules fullerene $\mathrm{C}_{60}, 1$ and 2 .

Nonlinear absorption and refraction were studied for both new compounds. The optical limiting process exhibited by 2.5 and $1.5 \mathrm{mM}$ solutions of the samples 1 and 2 , respectively, together with that of a $2.6 \mathrm{mM} \mathrm{C}_{60}$ toluene solution, had the same linear transmittance of $65 \%$ at $532 \mathrm{~nm}$ (Fig. 4). As it can be seen in Fig. 4, $\mathrm{C}_{60}$ is exhibiting stronger optical limiting efficiency than the ligand 1 and the complex 2, while having relatively higher efficiency than the ligand. It should be noted that nonlinear optical absorption was observed, while nonlinear optical refraction remained negligible (hidden), for both the ligand and the complex. The comparison between the nonlinear optical absorption of the two compounds indicates that the ligand has a lower NLO absorption than the corresponding $\mathrm{Zn}$ (II) complex. In addition, the optical efficiency of optical limiting of the ligand is higher than the $\mathrm{Zn}(\mathrm{II})$ complex. Therefore we find that these molecular structures are important for applications in photonics and optoelectronics.

Figure 4 reveals that the ligand has a larger optical limiting efficiency than its complex. There are several factors which are responsible for the optical limiting efficiency of fullerene derivatives. We may mention the efficient population transfer of the first excited singlet state population to the triplet state occurring on the nanosecond scale. Indeed, as observed in Fig. 2, the complex presents a broadening of the UV-vis spectra.

This interesting result showing the beneficial role played by the metal cation will incite us to explore other systems. In particular, we may proceed to study an electronic exchange between donor/acceptor groups to further improve the nonlinearity for other photonic applications.

In conclusion, the optical limiting behavior of an electroactive iminopyridine ligand was investigated. We have demonstrated that these molecular structures are important for applications in photonics and optoelectronics. This study opens up new perspectives for optoelectronic applications while controlling the nonlinear optical parameters. Thus, the imino-pyridine-TTF derivatives are good optical limiters and are interesting materials for nonlinear optics. This depends mainly on the type of intramolecular between the donor and acceptor units and on the effect of the coordinated metal cation. In the future, other p-conjugated molecular systems will be prepared to increase the optical parameters.

M.A.K and B.S. acknowledge support in Poland from the National Science Centre under grant agreement DEC2012/06/M/ST2/00479.

\section{References}

[1] V. Rosso, J. Loicp, Y. Renotte, Y. Lion, J. Non-Crystalline Solids 342 (1-3), 140 (2004).

[2] L. Jiang, Q. Chang, Q.Y. Ouyang, H.B. Liu, Y.X. Wang, X.R. Zhang, et al., Chemical Phys, 324(2-3), 556 (2006).

[3] B. Sahraoui, X. Nguyen Phu, M. Sallé, A. Gorgues, Opt. Lett. 23(23), 1811 (1998).

[4] A. Apostoluk, D. Chapron, C. Fiorini-Debuisschert, G. Gadret, J.M. Nunzi, P. Raimond, B. Sahraoui, Opt. Lett. 27(22), 2028 (2002).

[5] Ts. Kolev, I.V. Kityk, J. Ebothe, B. Sahraoui, Chem. Phys. Lett. 443(4 6 ), 309 (2007).

[6] K. Iliopoulos, A. El-Ghayoury, B. Derkowska, A. Ranganathan, P. Batail, D. Gindre, B. Sahraoui, Appl.Phys. Lett. 101, 261105 (2012).

[7] K. Iliopoulos, A. El-Ghayoury, H. El Ouazzani, M. Pranaitis, E. Belhadj, E. Ripaud, M. Mazari, M. Sallé, D. Gindre, B. Sahraoui, Opt. Expr. 20, 25311 (2012)

[8] H. El Ouazzani, K. Iliopoulos, M. Pranaitis, O. Krupka, V. Smokal, A. Kolendo, B. Sahraoui, J. Phys. Chem. B 115, 1944 (2011)

[9] F. Kajar, J. M. Nunzi, Functional Organic and Polymeric Materials (John Wiley and Sons Ltd., New York, 2000).

[10] K. Iliopoulos, I. Guezguez, A.P. Kerasidou, A. El-Ghayoury, D. Branzea, G. Nita, N. Avarvari, H. Belmabrouk, S. Couris, B. Sahraoui, Dyes and Pigments 101, 229 (2014).

[11] G. Nita, D. Branzea, F. Pop, A. El-Ghayoury, N. Avarvari, Crystals 2, 338 (2012).

[12] E. Coronado, J.R. Galán-Mascarós, C.J. Gómez-García, V. Laukhin, Nature 408, 447 (2000)

[13] M. Nihei, N. Takahashi, H. Nishikawa, H. Oshio, Dalton Trans 40, 2154 (2011).

[14] G.J.P. Britovsek, V.C. Gibson, B.S. Kimberley, P.J. Maddox, S.J. McTavish, G.A. Solan, et al., Chem. Commun. 849, 50 (1998).

[15] B.L. Small, M. Brookhart, A.M.A. Bennett, J. Am. Chem. Soc. 120, 4049 (1998).

[16] K. Iliopoulos, S. Couris, U. Hartnagel, A. Hirsch, Chem. Phys. Lett. 448, 243 (2007).

[17] N. Liaros, K. Iliopoulos, M.M. Stylianakis, E. Koudoumas, S. Couris, Opt. Mat. 36, 112 (2014). 